\title{
INTERFAZ CEREBRO-ORDENADOR PARA EL CONTROL DE UNA SILLA DE RUEDAS A TRAVÉS DE DOS PARADIGMAS DE NAVEGACIÓN
}

\author{
Álvaro Fernández-Rodríguez, Francisco Velasco-Álvarez y Ricardo Ron-Angevin \\ Dpto. Tecnología Electrónica, ETSI Telecomunicación, Universidad de Málaga \\ afernandezrguez@uma.es, \{rron, fvelasco\}@dte.uma.es
}

\begin{abstract}
Resumen
Una interfaz cerebro ordenador (BCI, de braincomputer interface) es una tecnología que permite a pacientes con disfunciones motoras severas usar sus señales electroencefalográficas para crear un canal de comunicación y controlar ciertos dispositivos. En este artículo será estudiada la aplicación de dos paradigmas para controlar una silla de ruedas mediante un sistema BCI: modo continuo $e$ interruptor. Cinco usuarios sanos tomaron parte del estudio, de los cuales cuatro controlaron la silla de ruedas. Los resultados mostraron que el modo de control interruptor obtuvo un mejor rendimiento para los tramos de avance, mientras que el modo continuo fue más adecuado para mantener la silla de ruedas detenida. Esto podría indicar que un modo de control híbrido con estas ventajas podría ser el objetivo de futuras propuestas.
\end{abstract}

Palabras Clave: interfaz cerebro-ordenador (BCI), silla de ruedas, navegación, control.

\section{INTRODUCCIÓN}

Enfermedades como la esclerosis lateral amiotrófica (ELA) o lesiones del tronco encefálico pueden dar lugar a un deterioro de las funciones motoras de los pacientes afectados, lo que podría provocarles la necesidad de utilizar tecnología asistencial para mejorar su calidad de vida. Sin embargo, en algunos casos, la severa reducción de sus funciones motoras les puede llegar a impedir el uso de sistemas convencionales dependientes de dichas funciones, como las necesarias para manejar un joystick o incluso un eye-tracker. Por lo tanto, la solución podría residir en la utilización de sistemas que no requieran la capacidad motora del usuario para controlarlos. Precisamente, las interfaces cerebrocomputadora (BCI, de brain-computer interface) corresponden a una tecnología que permite usar las señales electroencefalográficas (EEG) de los pacientes para crear un canal de comunicación entre ellos y el dispositivo que desean controlar sin necesidad de control muscular. Estos sistemas se han implementado en el desarrollo de dispositivos tales como una matriz de letras para escribir [2], un sistema de control domótico [1] o una silla de ruedas (BCW, de brain-controlled wheelchairs) [6]. Concretamente, estudiar el control de una silla de ruedas será el objetivo del presente trabajo.

Desde la publicación de la primera silla de ruedas en 2005 por [10], se han realizado numerosas propuestas sobre diferentes aspectos del dispositivo, como la señal EEG registrada, las tareas que el usuario debe realizar, las propiedades específicas de la interfaz o el sistema de navegación implementado [3].

La presente propuesta, tal y como han hecho la mayoría de BCWs presentadas hasta la fecha, se ha basado en la señal EEG más utilizada para el control de una BCW en entornos reales, los ritmos sensorimotores (SMR, de sensorimotor rhythm) [3]. Esta señal se basa en el fenómeno de la (de)sincronización asociada a eventos (ERD/ERS, de event-related (des)synchronization) y que origina cambios de amplitud producidos en el área de la corteza sensoriomotora en la banda $\mu(7-13 \mathrm{~Hz})$ al realizar una tarea de imaginación motora (IM). De este modo, dicha señal puede ser modulada por el propio usuario para ser utilizada en un sistema BCI [7].

La taxonomía principal para clasificar las BCWs según el método de navegación empleado los divide entre sillas de bajo y alto nivel. Por un lado, en la navegación de bajo nivel, el control del dispositivo se logra mediante comandos simples de navegación, tales como 'avanzar' o 'girar a la derecha'. Por otro lado, la navegación de alto nivel permite a los usuarios ejecutar comandos como 'llévame a la cocina' o 'abandona esta habitación'. A pesar de que la navegación de alto nivel podría provocar una menor carga de trabajo y resultar más cómoda, ya que el usuario simplemente debe seleccionar los destinos, la presente propuesta se enmarca dentro de los sistemas de bajo nivel. Esto es debido a que dichos sistemas podrían resultar más apropiados para entornos incontrolados en los que la silla de ruedas deba poseer una adecuada flexibilidad para evitar posibles obstáculos o adaptar su trayectoria a nuevas 
modificaciones en el entorno. Asimismo, dentro de los sistemas de bajo nivel se pueden establecer más divisiones basándose en el tipo de desplazamiento de la silla de ruedas, destacando el control discreto y continuo. En primer lugar, en el control discreto, la selección de un comando de navegación implica un movimiento prefijado como un giro de $45^{\circ}$ o un avance de $1 \mathrm{~m}$. En segundo lugar, en el control continuo, el usuario puede controlar la extensión del movimiento tras la selección de un comando de navegación como, por ejemplo, la amplitud del giro o la longitud del avance. En este tipo de manejo, lo normal es que el movimiento continúe siempre y cuando el usuario mantenga activo el comando.

El grupo BCI de la Universidad de Málaga (UMA$\mathrm{BCI})$ ha realizado una propuesta de control alternativa a las descritas anteriormente sobre el control de una silla de ruedas y un robot móvil en entornos virtuales: el paradigma de modo interruptor [11], [9]. En este paradigma, el usuario tiene que realizar la tarea correspondiente (por ejemplo, IM) para cambiar el estado de la silla de ruedas, es decir, realizar un desplazamiento o detener el movimiento, mientras que si el usuario quiere permanecer en el estado actual, es decir, continuar el desplazamiento o mantener la posición, tiene que ejecutar una tarea alternativa. Este control comparte con el paradigma continuo que el usuario puede controlar la distancia exacta de sus desplazamientos. Sin embargo, la principal diferencia entre el paradigma propuesto y el continuo es que, en este último, el usuario debe tener la capacidad de mantener ambas tareas de forma estable: una tarea para seleccionar el comando activo (por ejemplo, avanzar) y otra tarea para permanecer en reposo. Sin embargo, en el modo interruptor, el usuario debe tener la capacidad de realizar una tarea rápidamente (asociada al cambio de estado en el desplazamiento de la silla de ruedas), pero tener un control estable en la otra (asociada al mantenimiento del estado actual de la silla de ruedas). El funcionamiento de estos paradigmas se explicará con más detalle en la sección 2.3. Aplicación de navegación.

En definitiva, el objetivo del presente estudio es evaluar los paradigmas de control continuo e interruptor en una tarea de navegación básica con una BCW.

\section{MÉTODO}

\subsection{PARTICIPANTES}

Cinco usuarios sanos formaron parte del estudio (edad media de 19,75 $\pm 0,5$ años; cuatro hombres, una mujer), estudiantes de la Universidad de Málaga. Ninguno tenía experiencia previa en el manejo de estos sistemas y fueron captados a través del uso de publicidad en las redes sociales y el boca a boca, usando como reclamo el poder controlar una silla de ruedas con la mente y una recompensa económica de entre 5 y $10 €$ por participar, dependiendo de si completaban la primera fase de calibración del estudio. El experimento fue aprobado por el Comité Ético de Experimentación de la Universidad de Málaga y cumplió los criterios de la Declaración de Helsinki. Los participantes declararon en la hoja de consentimiento informado no tener ningún historial médico de desórdenes neurológicos o psiquiátricos, así como no tomar algún tipo de medicación de forma regular. Como criterio del diseño, en la tarea de calibración se estableció un límite máximo de error del $30 \%$ para poder continuar en el estudio. Este límite, anteriormente utilizado en [4], fue establecido para garantizar que los usuarios que pasaran a la siguiente prueba tuvieran un control aceptable de su señal SMR, la cual sería empleada para controlar la BCW.

\subsection{ADQUISICIÓN DE DATOS Y PROCESAMIENTO DE LA SEÑAL}

Las señales EEG fueron registradas con una frecuencia de muestro de $200 \mathrm{~Hz}$ a través de dos canales laplacianos grandes (para más detalles, ver [5]), situados sobre las áreas somatosensoriales motoras izquierda y derecha utilizando la siguiente configuración de electrodos: C3, F3, P3, T7 y Cz para el canal laplaciano izquierdo, y C4, F4, P4, T8 y $\mathrm{Cz}$ para el canal laplaciano derecho. Los electrodos de tierra y referencia fueron situados en las posiciones $\mathrm{AFz}$ y $\mathrm{Fz}$, respectivamente. Esta configuración fue realizada de acuerdo al sistema internacional 10/20. La señal fue amplificada con un amplificador actiCHamp (Brain Products $\mathrm{GmbH}$, Munich, Germany). No fue utilizada ninguna técnica de detección de artefactos.

Como se mencionó anteriormente, los usuarios participaron en una fase de entrenamiento para proceder a la calibración del sistema (ver 2.5.1 Calibración). En esta tarea, se registró la señal EEG de cada participante llevando a cabo dos tareas mentales durante numerosos ensayos. Con estos datos, se calculó la banda reactiva de cada usuario y su error relacionado. Después de procesar la señal de cada participante, se seleccionaron aquellos con un error en la clasificación inferior al 30\%, guardándose sus parámetros para ser utilizados en la posterior tarea con feedback. El procesado de los datos y la generación del feedback fue llevada a cabo por MATLAB y se basó en el procedimiento detallado en [8] que se explicará a continuación:

a. Obtención de la banda de frecuencia reactiva. La banda de frecuencia reactiva de cada participante fue automáticamente seleccionada 
entre todas las posibles combinaciones de entre 5 y $17 \mathrm{~Hz}$ (con un mínimo de ancho de banda de 2 $\mathrm{Hz}$ ). La búsqueda de la banda de frecuencia óptima fue limitada a la banda $\mu$ por motivos de simplicidad. Para cada intervalo de frecuencia probado, era llevado a cabo un procedimiento de extracción de características y clasificación que daba lugar a la obtención del porcentaje de error obtenido por el usuario en cada banda de frecuencia. Aquella banda de frecuencia con el mínimo error en la clasificación era la seleccionada como su correspondiente banda de frecuencia reactiva.

b. Extracción de características. La extracción de características consistió en la estimación de la potencia media de la señal en cada uno de los dos canales EEG en el intervalo específico de frecuencia. Esta media era calculada a través de: i) la digitalización de la señal EEG y un filtrado paso banda tipo Butterworth de quinto orden, ii) el cálculo del cuadrado de cada muestra resultante $y$ iii) el promediado de las consecutivas 100 muestras, dando una estimación de la potencia de banda para intervalos de $500 \mathrm{~ms}$.

c. Clasificación. Las características de cada uno de los canales para todos los ensayos fueron usadas para calcular el porcentaje de error para cada instante de tiempo posible a través de un análisis lineal discriminante (LDA, de linear discriminant analysis) y un promediado mediante validación cruzada de 10 iteraciones. De esta forma, se obtenía el porcentaje de error mínimo estimado para las características de potencia de la banda de frecuencia determinada.

d. Generación del feedback. La banda de frecuencias anteriormente mencionada y sus características eran utilizadas para configurar el LDA, cuya clasificación resultante determinaba el valor del feedback 'L'. Este feedback era actualizado cada $31,25 \mathrm{~ms}$.

\subsection{APLICACIÓN DE NAVEGACIÓN}

Puesto que el propósito de este estudio es realizar una comparación preliminar entre dos métodos de navegación, se ha decidido simplificar la interfaz de manera que el usuario sólo pudiera seleccionar un comando activo de navegación: avanzar con la BCW. De esta manera, además, queda eliminado cualquier otro factor de la interfaz que pudiera sesgar la comparación.

Han sido puestos a prueba dos modos de control, modo continuo y modo interruptor, sin embargo, el paradigma de selección del comando activo ha sido similar en ambas interfaces. La tarea activa que fue utilizada como comando de control era la IM motora de mano derecha, siendo la tarea alternativa la realización de algún tipo de operación mental que fuera capaz de mantener al usuario concentrado y sin pensar en la tarea de IM (por ejemplo, cálculo matemático). Cuando el participante llevaba a cabo la tarea de IM, provocaba un estiramiento de una barra - distancia 'L', oculta al usuario - como resultado de la clasificación del LDA cada 31,25 ms. En caso de que el clasificador determinase que la tarea mental era IM de mano derecha, la barra se extendía; de otra manera, si el clasificador determinaba que el participante se encontraba realizando la tarea alternativa, la barra permanecía inmóvil y en un tamaño reducido. En caso de que el usuario desease realizar una selección, éste debía mantener la barra por encima de un 'umbral de selección' y durante un tiempo superior a un 'tiempo de selección' (alrededor de $1 \mathrm{~s}$ ). Si la distancia ' $L$ ' de la barra descendía por debajo de un 'umbral de selección' durante un tiempo menor que un 'tiempo de reinicio' (alrededor de 0,5 s), el 'tiempo de selección' continuaba acumulándose y no era reiniciado. Los parámetros de 'tiempo de selección' y 'tiempo de reinicio', así como 'el umbral de selección', podían ser configurados manualmente para facilitar o dificultar la capacidad de selección de los usuarios. A partir de este punto, lo que suceda dependerá del tipo de control del que disponga la silla de ruedas.

Por un lado, en el modo continuo, la tarea de IM de mano derecha serviría para mover la $\mathrm{BCW}$, es decir, cuando el usuario realizaba dicha tarea, extendería la barra anteriormente mencionada y el dispositivo avanzaría de forma continuada siempre que la barra se encontrase por encima del 'umbral de selección'. En cambio, para detener o mantener la BCW en reposo, el usuario debería realizar la tarea alternativa y mantener la barra por debajo de dicho umbral.

Por otro lado, con respecto al modo interruptor, la tarea de IM de mano derecha serviría para cambiar el estado de la silla, como si de un interruptor convencional se tratase. En caso de que el usuario se encontrase en reposo y quisiera mover la silla, debería llevar a cabo la tarea de IM y, cuando empezase a moverse, cambiar a la tarea alternativa para continuar el movimiento. Una vez en movimiento, si el usuario desease detener la silla, simplemente debería volver a ejecutar la tarea de IM.

\subsection{SILLA DE RUEDAS ROBÓTICA}

La BCW utilizada consistió en una modificación de una silla eléctrica Invacare Mistral3 equipada con una placa de control personalizada que emulaba el joystick de la silla de ruedas (figura 1). Dicha placa de control permitía el control de la silla de ruedas en 
tiempo real y recibía información de dos codificadores rotatorios a través de un bus I2C. Además, era conectada a través de un puerto USB a la aplicación de control escrita en $\mathrm{C}$ que era ejecutada en el ordenador portátil. Esta aplicación recibía, vía conexión TCP, los comandos de navegación seleccionados por la aplicación ejecutada en MATLAB. Los dos codificadores rotatorios magnéticos AS5048 fueron colocados en las ruedas para garantizar, a través de un sistema de odometría, que la silla no se desviaba de su trayectoria en ningún momento. La velocidad del dispositivo era de aproximadamente $5,5 \mathrm{~s}$ para cada metro de avance $(0,18 \mathrm{~m} / \mathrm{s})$.

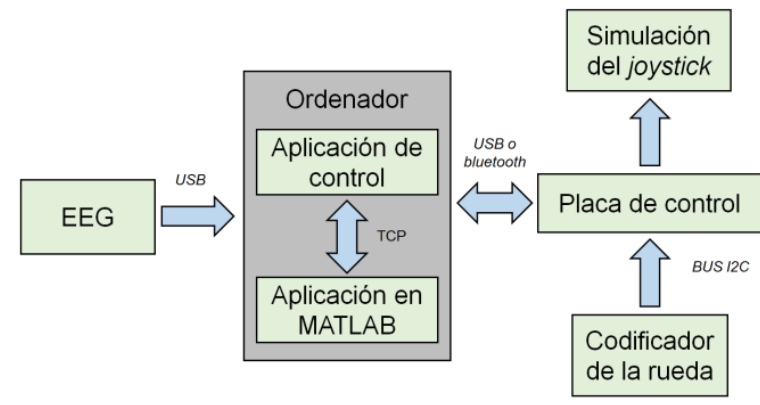

Figura 1. Esquema del sistema de control de la silla de ruedas.

\subsection{PROCEDIMIENTO}

El estudio fue llevado a cabo en una sola sesión de una duración total aproximada de dos horas y constaba de dos partes: i) una prueba inicial de calibración y ii) una tarea de control con la $\mathrm{BCW}$ (figura 2). Tanto la tarea de calibración como la de navegación fueron llevadas a cabo en una habitación espaciosa y sin tráfico de gente de la Escuela Técnica Superior de Ingeniería de Telecomunicación de la Universidad de Málaga. Una vez el usuario llegaba a la zona de realización del experimento, se le explicaba con detalle en qué consistiría la prueba, firmaba la hoja de consentimiento informado y se procedía a la preparación de la prueba, incluyendo el montaje del EEG. Todo este proceso de preparación y montaje, previo al comienzo de la prueba, tenía una duración aproximada de $30 \mathrm{~min}$.

\subsubsection{Calibración}

El participante era sentado en la BCW frente a un ordenador portátil de 15,6 pulgadas. La tarea que debía llevar a cabo consistía en la realización de dos tareas mentales para que el sistema acabase reconociendo los cambios producidos en la actividad cerebral del usuario cuando imaginaba cada una de estas tareas, con el fin de que posteriormente pudieran ser utilizadas como comandos de control en

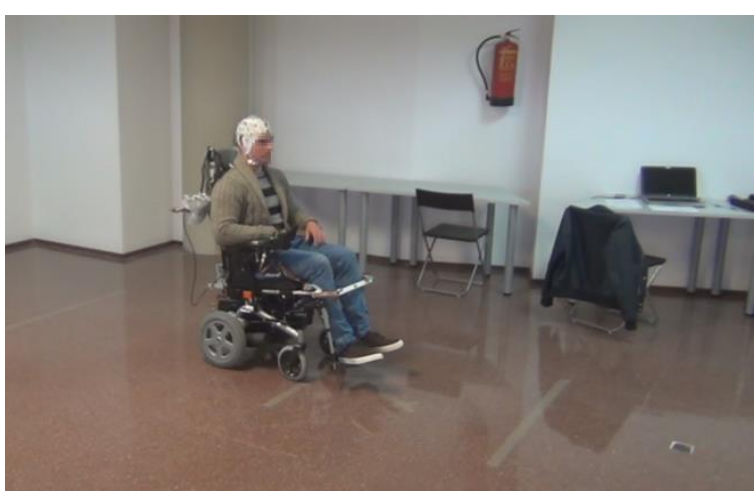

Figura 2. Participante durante la ejecución de la tarea de control con la silla de ruedas.

la BCW. El paradigma de calibración utilizado ha sido presentado en anteriores experimento del grupo UMA-BCI [8]. Concretamente, la prueba consistía en lo siguiente: i) el usuario veía un coche estacionado en el medio de una carretera que, al comenzar el ensayo, arrancaba el motor, ii) tras $2 \mathrm{~s}$, el coche empezaba a avanzar, dando lugar a la posible aparición de un charco de agua en el lado izquierdo de la pantalla que llegará a estar situado al lado del coche a partir de 4,25 s hasta el final del ensayo. En caso de que el charco de agua fuera presentado, el participante debía ejecutar la tarea de IM de mano derecha desde que comenzaba a ver el charco hasta que el sonido del motor del coche se apagase; si por el contrario, el charco no aparecía, el usuario debía concentrarse en realizar la tarea alternativa durante todo ese tiempo, es decir, en el intervalo de tiempo de 2 a 8 s (figura 3). La tarea fue dividida en cuatro bloques de 40 ensayos - 20 de IM y 20 de tarea alternativa, ordenados aleatoriamente - con el fin de no fatigar al usuario y que pudiera descansar entre bloques. Asimismo, el participante disponía de un breve descanso aleatorio de 0,5-3 s entre ensayos para poder acomodarse en la silla o realizar cualquier otro movimiento que no debiera realizar durante el ensayo.

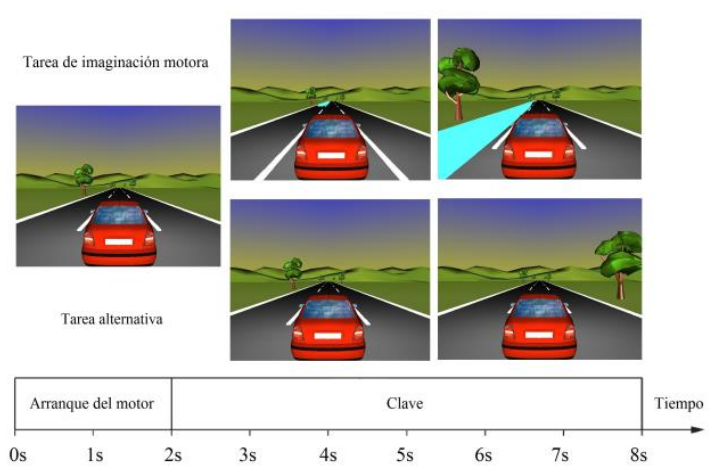

Figura 3. Temporalización de los ensayos de la tarea de calibración 
La tarea tenía una duración aproximada de media hora, excluyendo el tiempo de descanso entre bloques. Los datos obtenidos de esta fase eran procesados posteriormente para la obtención de la banda de frecuencia reactiva, el porcentaje de error en la clasificación y los parámetros óptimos del clasificador. Como se mencionó anteriormente, fueron excluidos aquellos participantes que en esta tarea tuvieron una tasa de error en su banda de frecuencia reactiva superior al $30 \%$. El entorno de esta tarea virtual fue desarrollado con VRML 2.0.

\subsubsection{Tarea de control con la $\mathrm{BCW}$}

Aquellos participantes que completaron la tarea de calibración con un porcentaje de error inferior al $30 \%$ continuaron en el experimento para poder controlar la $\mathrm{BCW}$. La tarea de navegación a realizar consistió en un tramo recto de un total de $8,4 \mathrm{~m}$ en el cual el usuario debía realizar tres secciones de avance de 2 $\mathrm{m}$ y dos de parada de 1,2 m (figura 4). El objetivo del participante fue completar los tramos de avance en el menor tiempo posible mientras que en los tramos de parada debía detener la $\mathrm{BCW}$ hasta un máximo de 60 $\mathrm{s}$, no siendo necesario que realizara este tiempo de parada de una sola vez. Antes de realizar la tarea, el usuario disponía de un tiempo de entrenamiento manejando la BCW (alrededor de 10 minutos para cada tipo de control), de forma que quedase asegurado que comprendía el paradigma, pudiera practicar un poco y se acostumbrase a su desplazamiento.

Debido a que el participante era instruido para que permaneciera inmóvil durante la prueba, éste recibía determinadas indicaciones acústicas para saber cuándo entraba en cada tramo. Concretamente, cuando el usuario se encontraba aproximadamente a $40 \mathrm{~cm}$ de la zona de parada, se le indicaba por voz la palabra «llegando», mientras que la palabra utilizada una vez entraba a la zona de parada fue «dentro». Asimismo, si el usuario realizaba correctamente los $60 \mathrm{~s}$ dentro de dicho intervalo, recibía la orden «tiempo, continúa», indicándole que completó exitosamente el minuto de parada y que podía continuar el recorrido. Por el contrario, si el usuario abandonaba la zona de parada antes de los $60 \mathrm{~s}$, se le indicaba mediante la orden «fuera, continúa» que debía completar el siguiente tramo de avance lo más rápido posible.

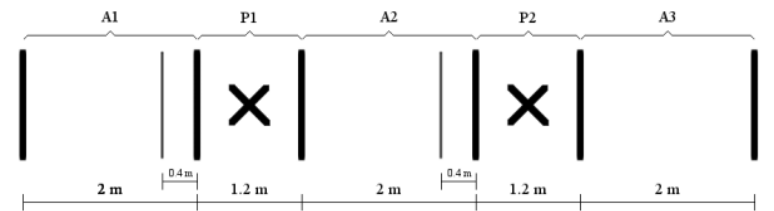

Figura 4. Recorrido de la tarea de navegación a completar con la silla de ruedas.

\subsection{EVALUACIÓN}

Además de las variables relativas a los tiempos y a las selecciones deseadas frente a las no deseadas, para conocer el rendimiento del usuario de forma general, se han creado dos variables que resumen la ejecución del participante para las secciones de avance y parada:

$$
\text { Ratio de tiempo de avance }=\frac{A t_{\text {min }}}{A t_{o}}
$$

Siendo $A t_{\min }$ el tiempo mínimo requerido para completar los tramos de avance (11 s) y $A t_{o}$ el tiempo observado, es decir, el ejecutado por el usuario.

$$
\text { Ratio de tiempo de parada }=\frac{P t_{o}}{P t_{\text {máx }}}
$$

Siendo $\mathrm{Pt}_{\text {máx }}$ el tiempo máximo objetivo para completar los tramos de parada (60 s) y $\mathrm{St}_{0}$ el tiempo observado, es decir, el ejecutado por el usuario.

Estas ecuaciones recaen en la idea de que un buen rendimiento será aquel en el cual el usuario realice el mayor tiempo de parada posible (nunca superior a 60 s) y menor tiempo de avance (nunca inferior a $11 \mathrm{~s}$, que es lo que tarda la silla en recorrer $2 \mathrm{~m}$ ). De esta manera, la ecuación 1 registrará el rendimiento de los usuarios en los avances, mientras que la ecuación 2 lo hará en las paradas. Los valores de ambas ecuaciones indicarán mejores resultados cuanto más cercanos sean a uno.

\section{RESULTADOS}

\subsection{CALIBRACIÓN}

La banda de frecuencia reactiva y el error mínimo obtenido en la clasificación de cada usuario son mostrados en la tabla 1 . De media, el mínimo error obtenido fue $20,38 \pm 8,08 \%$. De los cinco participantes, sólo uno (S2) tuvo una tasa de error superior al punto de corte (30\%) y fue eliminado del estudio.

\subsection{TAREA DE NAVEGACIÓN}

La tabla 2 muestra el tiempo que cada usuario estuvo ejecutando cada una de las dos tareas, así como el número de comandos de avance ejecutados, en cada sección del recorrido. Por tanto, de ella puede ser extraído el tiempo medio que emplearon los usuarios para completar un tramo de avance, el cual fue de $26,42 \pm 5,85 \mathrm{~s}$ para el modo continuo y de $12,75 \pm 0,83$ $\mathrm{s}$ para el modo en interruptor. De igual manera, este 
Tabla 1: Resultado de los participantes en la tarea de calibración.

\begin{tabular}{ccc}
\hline Usuario & $\begin{array}{c}\text { Banda de frecuencia } \\
\text { reactiva(Hz) }\end{array}$ & $\begin{array}{c}\text { Error mínimo } \\
(\%)\end{array}$ \\
\hline S1 & $7-17$ & 8,81 \\
S2 & $13-16$ & 31,31 \\
S3 & $12-14$ & 18,44 \\
S4 & $5-15$ & 20,94 \\
S5 & $11-15$ & 22,38 \\
Media & $9,6 \pm 3,44-15,4 \pm 1,14$ & $20,38 \pm 8,08$ \\
\hline
\end{tabular}

tiempo medio puede ser calculado para las secciones de parada, ofreciendo como resultado un tiempo medio de $53,25 \pm 12,84 \mathrm{~s}$ y $22,5 \pm 14,46 \mathrm{~s}$ para los paradigmas de continuo e interruptor, respectivamente. Por otro lado, el número medio de comandos de avance requeridos para para completar los tramos de avance fueron de 3,91 $\pm 1,1$ y 0,92 $\pm 0,32$ para los modos continuo e interruptor, respectivamente. Nótese que el valor del número de comandos para el modo interruptor es incluso inferior a la unidad, lo que significa que algunas veces la silla realizó el tramo de avance sin realizar ninguna selección de avance; hay que tener en cuenta que si la silla no realiza ninguna parada desde que entra al tramo de avance, no es necesario que dicho comando sea seleccionado. Asimismo, estos valores en los tramos de parada fueron de $2,88 \pm 1,8$ selecciones no deseadas del comando de avance en el modo continuo y $1,13 \pm 0,48$ en el modo interruptor.

Finalmente, en cuanto a las medidas presentadas en el apartado 2.6 Evaluación relativas a los ratios de tiempo de avance y parada, podemos observar una tendencia, según la cual, el modo en interruptor ha resultado más eficaz en los tramos de avance, mientras el modo continuo ha realizado considerablemente mejor los tramos de parada (figura 4).

\section{CONCLUSIONES}

Los resultados del estudio sugieren que cada modo de control posee características específicas que dan lugar a ciertas ventajas e inconvenientes que deben ser tenidos en cuenta a la hora de ser utilizados. Concretamente, en este estudio preliminar hemos observado una tendencia del modo interruptor a ofrecer un mejor rendimiento que el modo continuo en los tramos de avance, ya que el usuario podía recorrer una mayor distancia con una única selección de comando. No obstante, esta ventaja ha mostrado la posibilidad de ser un inconveniente en los tramos de parada, donde el modo continuo ha ofrecido mejores resultados.

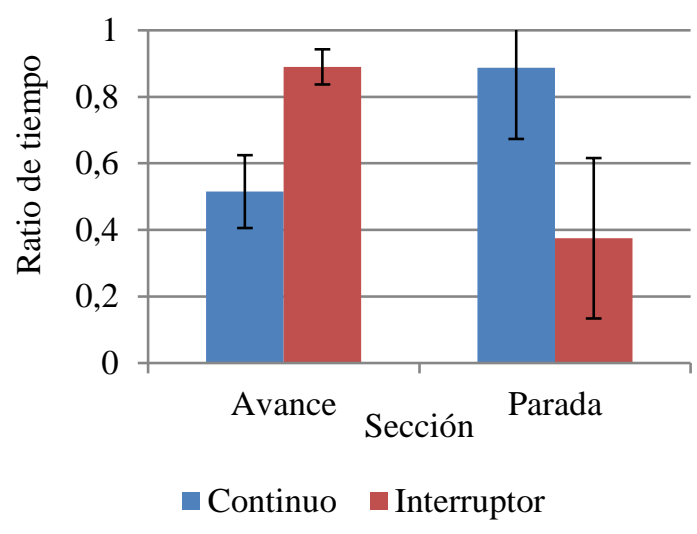

Figura 4. Variables de ratios de tiempo para los tramos de avance (ecuación 1) y los tramos de parada (ecuación 2) para ambos modos de control.

Tabla 2. Variables de rendimiento recogidas durante la tarea de navegación con la silla de ruedas.

\begin{tabular}{|c|c|c|c|c|c|c|c|c|c|c|c|c|c|c|c|c|}
\hline \multirow{3}{*}{ Usuario } & \multirow{3}{*}{ Manejo } & \multicolumn{10}{|c|}{ Tiempo (s) } & \multicolumn{5}{|c|}{ Selecciones de avance } \\
\hline & & \multicolumn{2}{|c|}{ A1 } & \multicolumn{2}{|c|}{$\mathrm{P} 1$} & \multicolumn{2}{|c|}{$\mathrm{A} 2$} & \multicolumn{2}{|c|}{$\mathrm{P} 2$} & \multicolumn{2}{|c|}{ A3 } & \multirow{2}{*}{ A1 } & \multirow{2}{*}{$\mathrm{P} 1$} & \multirow{2}{*}{$\mathrm{A} 2$} & \multirow{2}{*}{$\mathrm{P} 2$} & \multirow{2}{*}{ A3 } \\
\hline & & $\mathrm{T}_{\mathrm{a}}$ & $\mathrm{T}_{\mathrm{p}}$ & $\mathrm{T}_{\mathrm{a}}$ & $\mathrm{T}_{\mathrm{p}}$ & $\mathrm{T}_{\mathrm{a}}$ & $\mathrm{T}_{\mathrm{p}}$ & $\mathrm{T}_{\mathrm{a}}$ & $\mathrm{T}_{\mathrm{p}}$ & $\mathrm{T}_{\mathrm{a}}$ & $\mathrm{T}_{\mathrm{p}}$ & & & & & \\
\hline \multirow{2}{*}{$\mathrm{S} 1$} & continuo & 16 & 5 & 4 & 56 & 11 & 4 & 7 & 1 & 13 & 21 & 5 & 3 & 2 & 1 & 5 \\
\hline & interruptor & 11 & 0 & 7 & 26 & 10 & 2 & 8 & 43 & 13 & 5 & 1 & 1 & 1 & 2 & 2 \\
\hline \multirow{2}{*}{ S3 } & continuo & 13 & 4 & 6 & 54 & 11 & 0 & 2 & 58 & 16 & 17 & 2 & 2 & 0 & 1 & 5 \\
\hline & interruptor & 11 & 0 & 8 & 14 & 10 & 0 & 7 & 2 & 12 & 2 & 1 & 1 & 0 & 1 & 1 \\
\hline \multirow{2}{*}{$\mathrm{S} 4$} & continuo & 16 & 6 & 12 & 48 & 16 & 18 & 7 & 53 & 18 & 11 & 4 & 8 & 5 & 3 & 5 \\
\hline & interruptor & 11 & 0 & 8 & 2 & 11 & 0 & 7 & 0 & 12 & 4 & 1 & 1 & 0 & 0 & 1 \\
\hline \multirow{2}{*}{ S5 } & continuo & 12 & 0 & 5 & 55 & 22 & 28 & 8 & 50 & 18 & 21 & 1 & 3 & 6 & 2 & 7 \\
\hline & interruptor & 11 & 0 & 8 & 26 & 11 & 4 & 8 & 6 & 11 & 2 & 1 & 1 & 1 & 2 & 1 \\
\hline
\end{tabular}

Cada uno de los tramos de avance del recorrido ha sido denominado, del primero al último, como A1, A2 y A3, mientras que las secciones primera y segunda de parada se denotaron como P1 y P2. Asimismo, para cada uno de estos tramos se ha registrado el tiempo que la silla ejecutaba cada uno de los comandos, los de avance $\left(\mathrm{T}_{\mathrm{a}}\right)$ y los de parada $\left(\mathrm{T}_{\mathrm{p}}\right)$. 
Para futuros estudios, sería conveniente volver a estudiar el control sobre los diferentes tipos de paradigmas con usuarios entrenados durante varias sesiones, ya que debe ser tenido en cuenta que todos los usuarios aquí presentados eran nuevos en el manejo de estas interfaces y el control de su señal EEG. Asimismo, podría resultar interesante estudiar la aplicación de nuevos paradigmas de navegación que consigan agrupar las ventajas de estos dos modos de control, de forma que el rendimiento del usuario y, por tanto, su experiencia durante el manejo resulte lo más adecuada posible.

\section{Agradecimientos}

Nos gustaría agradecer la asistencia de los participantes al estudio y la colaboración de Manon Bonnet en el diseño experimental y la realización de las pruebas. Este trabajo ha sido parcialmente financiado por el Ministerio de Economía y Competitividad y por los fondos Europeos FEDER a través del proyecto LICOM (DPI2015-67064R(MINECO/FEDER)), y la Universidad de Málaga.

\section{Referencias}

[1] Corralejo R., Hornero R. and Álvarez D., A Domotic Control System Using BrainComputer Interface (BCI), vol. 6691 LNCS, no. PART 1. 2011.

[2] Farwell L.A. and Donchin E., "Talking off the top of your head: toward a mental prothesis utilizing event-relatedpotencials," Electroencephalogr. Clin. Neurophysiol., vol. 70, no. 6, pp. 510-523, 1988.

[3] Fernández-Rodríguez Á., Velasco-Álvarez F. and Ron-Angevin R., "Review of real braincontrolled wheelchairs," J. Neural Eng., vol. 13, no. 6, p. 61001, 2016.

[4] Kubler A., Neumann N., Kaiser J., Kotchoubey B., Hinterberger Y. and N. Birbaumer, "Brain-computer communication: Self-regulation of slow cortical potentials for verbal communication," Arch. Phys. Med.
Rehabil., vol. 82, no. 11, pp. 1533-1539, 2001.

[5] McFarland D. J., McCane L. M., David S. V. and Wolpaw J. R., "Spatial filter selection for EEG-based communication," Electroencephalogr. Clin. Neurophysiol., vol. 103, no. 3, pp. 386-394, Sep. 1997.

[6] Millán J. D. R., Galán F., Vanhooydonck D., Lew E., Philips J. and Nuttin M., "Asynchronous non-invasive brain-actuated control of an intelligent wheelchair," Proc. 31st Annu. Int. Conf. IEEE Eng. Med. Biol. Soc. Eng. Futur. Biomed. EMBC 2009, vol. 2009, pp. 3361-3364, 2009.

[7] Pfurtscheller G., Brunner C., Schlögl A. and Lopes da Silva F. H., "Mu rhythm (de)synchronization and EEG single-trial classification of different motor imagery tasks," Neuroimage, vol. 31, no. 1, pp. 153159, 2006.

[8] Ron-Angevin R. and Díaz-Estrella A., "Brain-computer interface: Changes in performance using virtual reality techniques," Neurosci. Lett., vol. 449, no. 2, pp. 123-127, 2009

[8] Ron-Angevin R., Aurélie D., Yvan M., Lespinet-Najib V. and André J.M., "Braincomputer interface: comparison of two control modes to drive a virtual robot," Eur. Sci. J., vol. 7881, no. Im, pp. 177-189, 2015.

[10] Tanaka K., Matsunaga K. and Wang H. O., "Electroencephalogram-based control of an electric wheelchair," IEEE Trans. Robot., vol. 21, no. 4, pp. 762-766, 2005.

[11] Velasco-Álvarez F., Ron-Angevin R., Da Silva-Sauer L. and Sancha-Ros S., "Braincomputer interface: Comparison of two paradigms to freely navigate in a virtual environment through one mental task," 2010 5th Int. Conf. Broadband Biomed. Commun. IB2Com 2010, no. IB2Com 2010, pp. 1-5, 2010. 